\title{
Dose escalation of ripretinib can lead to response in advanced gastrointestinal stromal tumor patients refractory to the standard dose: a report of two cases
}

\author{
Philippos Apolinario Costa, Caroline Kamal Abdelmessih Hana, Navin Chakravarthy Balaji, \\ Anthony Frank Skryd, Brianna Nicole Valdes, Ramon O. Minjares, Priscila Barreto-Coelho, \\ Andrea P. Espejo-Freire, Muhammad O. Hakim, Emily Jonczak, Ty Subhawong, Alan Livingstone, \\ Jonathan C. Trent, Gina Z. D'Amato \\ University of Miami, Miami, FL, USA \\ Correspondence to: Philippos Apolinario Costa, MD. Internal Medicine Program, 1611 NW 12th Ave, Miami, FL 33136, USA. \\ Email: philippos500ac@hotmail.com.
}

\begin{abstract}
Despite an initial response, most metastatic gastrointestinal stromal tumor (GIST) patients will ultimately be refractory to all current therapies, including imatinib, sunitinib, and regorafenib. Ripretinib at $150 \mathrm{mg}$ daily was recently approved as an additional line of treatment. Few options remain after ripretinib. Here we report two cases with responses to dose escalation of ripretinib after progressing on the standard dose. Case 1: A 25-year-old man was diagnosed with a kit exon 9 mutated small intestine GIST after presenting with abdominal pain. The tumor was resected, but a year later, he developed metastases to the liver and pelvis. Over the course of three years, he received multiple lines of therapies as his disease progressed, including imatinib, sunitinib, and regorafenib. He was then placed on ripretinib $150 \mathrm{mg}$ daily. However, he had disease progression after two months. Ripretinib was increased to $150 \mathrm{mg}$ twice a day, which he tolerated well. After three months, he had regression of his disease. Case 2: A 54-year-old male was diagnosed with an unresectable kit exon 11 mutated gastric GISTs after presenting with abdominal pain. Imatinib led to an $80 \%$ regression, allowing surgical excision. A year later, his disease recurred. Over the course of 5 years, due to multiple recurrences, he received two additional surgeries, with imatinib, sunitinib, regorafenib, and avapritinib on either the adjuvant or neoadjuvant setting. Ultimately, he developed a metastatic GIST to the left suprarenal region, retroperitoneum, and epigastric region. He was then started on ripretinib $150 \mathrm{mg}$ daily, experiencing progression of his disease in three months. Ripretinib was escalated to $150 \mathrm{mg}$ twice a day, which he tolerated well, and after three months, he had a regression. Dose escalation of ripretinib leads to response in patients that progressed after the standard dose, and it is well-tolerated, being a promising new treatment option in advanced GIST.
\end{abstract}

Keywords: Ripretinib; gastrointestinal stromal tumor (GIST); INVICTUS, dose-escalation, case report

Received: 23 February 2021; Accepted: 20 April 2021; Published: 31 May 2021.

doi: $10.21037 /$ gist-21-1

View this article at: http://dx.doi.org/10.21037/gist-21-1

\section{Introduction}

Gastrointestinal stromal tumor (GIST) is the most common type of soft tissue sarcoma of the gastrointestinal (GI) tract, affecting 1.1 every 100,000 persons per year worldwide (1). Oncogenic driver mutations in the tyrosine kinase receptor c-kit gene are responsible for up to $80 \%$ of all GIST $(1,2)$, while mutations in platelet-derived growth factor receptor $\alpha(P D G F R A)$ gene are responsible for $5-10 \%(1,3)$. Those two mutations are mutually exclusive $(1,3)$. The remaining $5-10 \%$, so-called wild type, harbor a heterogeneous mix of mutations, including NF1, BRAF, SDH, and RAS (1). 

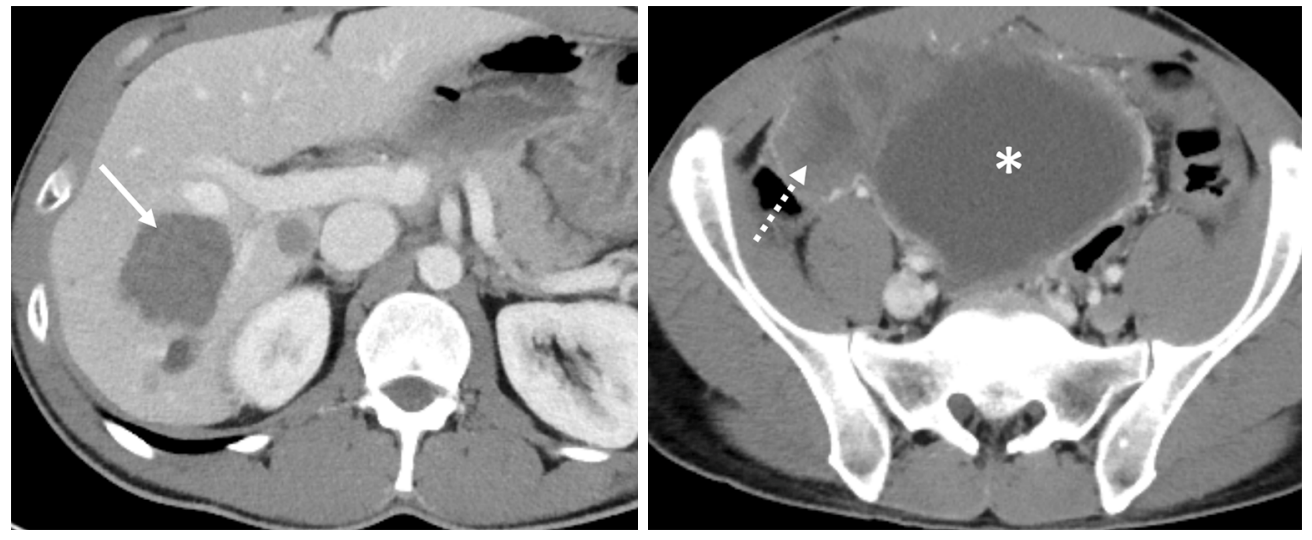

Figure 1 Axial contrast-enhanced CT in the portal venous phase demonstrated multiple metastases in the liver including a dominant hypodense right hepatic lobe mass (arrow), and a right lower quadrant peritoneal implant (dashed arrow).

The introduction of imatinib, a tyrosine kinase inhibitor (TKI) that inhibits KIT, dramatically changed GIST prognosis, leading to tumor control in $80 \%$ of cases (4). Despite imatinib, up to $90 \%$ of patients with metastatic GIST will ultimately be refractory to it, requiring further therapies (4). Although, $10 \%$ of patients can still benefit from Imatinib after 10 years of treatment (5). Regorafenib, a second-generation TKI, and sunitinib, a multi tyrosine kinase inhibitor, are currently used in refractory disease $(6,7)$. Nevertheless, the majority of patients will also eventually be refractory to these therapies (6). Understanding the need for new lines of treatments, ripretinib was approved in 2020 to be used in patients with advanced GIST refractory to three or more kinase inhibitors, including imatinib (4).

INVICTUS was the phase III trial that led to ripretinib approval. Patients with advanced GIST who had received at least 3 prior kinase inhibitors were randomly assigned to receive ripretinib at a dose of $150 \mathrm{mg}$ daily or placebo (4). In the ripretinib group, $51 \%$ of patients were progressionfree at 6 months (median progression-free survival of 6.3 months), whereas only $3.2 \%$ in the placebo group(4). Nevertheless, for ripretinib refractory patients, few options remain. The off-label use of a higher dose of ripretinib could be a valuable strategy in this scenario. Here we report two patients successfully treated with ripretinib dose escalation. We present the following cases in accordance with the CARE reporting checklist (available at http:// dx.doi.org/10.21037/gist-21-1).

\section{Case presentation}

All procedures performed in studies involving human participants were in accordance with the ethical standards of the institutional and/or national research committee(s) and with the Helsinki Declaration (as revised in 2013). Written informed consent was obtained from the patients.

\section{Case 1}

A 25-year-old man presented to a hospital with abdominal discomfort and pain. An abdominal computer tomography (CT) scan revealed a $12 \mathrm{~cm}$ bowel mass. The medical team performed an exploratory laparotomy with small bowel resection, and pathology showed a kit exon 9 mutated GIST. Due to the high-risk features of his disease, he was recommended adjuvant imatinib. However, the patient did not tolerate the medication and discontinued it. Follow up CT scan a year later showed multiple liver lesions, with biopsy confirming metastatic spread.

Due to exon 9 mutation, he was then started on imatinib $800 \mathrm{mg}$, which he took for a year until an abdominal CT scan showed increased liver lesions. He was then started on sunitinib, which he received for 6 months until disease progression. Regorafenib at $120 \mathrm{mg}$ dose was started. On regorafenib, he developed a grade 2 hand-feet syndrome that improved over time. Regorafenib was also given for 6 months until he developed disease progression (Figure 1).

He was placed on ripretinib $150 \mathrm{mg}$ daily, but two months later, he had disease progression in the liver and abdomen (Figure 2). Due to progression, dose escalation was proposed, and the patient tolerated well the dosage of $150 \mathrm{mg}$ twice per day, associated only with mild fatigue. His follow up CT scan three months after showed a $15 \%$ regression in his liver and abdominal lesions (Figure 3). He 

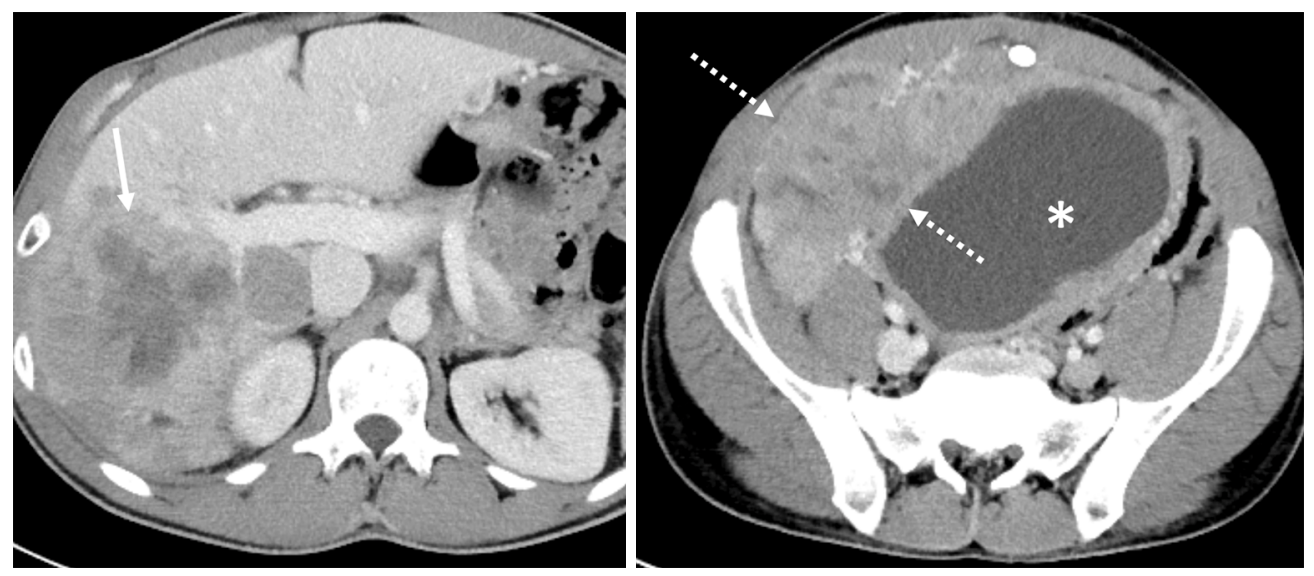

Figure 2 On subsequent CT three months after there has been marked interval progression in both the liver masses (arrow) and the peritoneal implants (dashed arrows). There is increased mass effect on the urinary bladder $\left(^{*}\right)$.
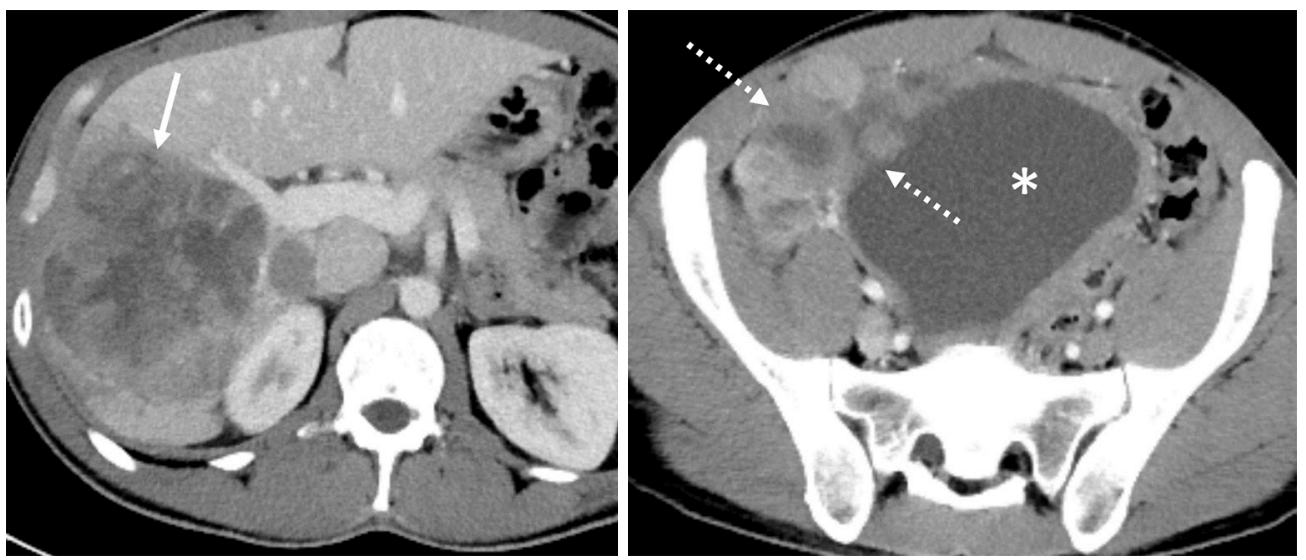

Figure 3 Follow-up CT three months after demonstrates decreased enhancement in the dominant right hepatic tumor (arrow) and reduction in the size of the adjacent smaller mass in the caudate lobe; additionally, decrease in size of the right lower quadrant implant (dashed arrows) indicate response to therapy.

remained progression free for 4 months.

\section{Case 2}

A 54-year-old male presented to the hospital with acute abdominal pain. At that time, he was found to have a $20 \mathrm{~cm}$ unresectable gastric KIT positive GIST. He was started on imatinib $400 \mathrm{mg}$ daily, obtaining an $80 \%$ regression in a year, allowing the lesion to be resected with negative margins. Due to high-risk features, imatinib was maintained.

Follow-up imaging a year later showed a recurrence in his transverse colon. He underwent a transverse colectomy and was placed on sunitinib postoperatively. The following year, CT scans showed a recurrence in the suprarenal region, which was resected with negative margins, followed by adjuvant imatinib at $800 \mathrm{mg}$ daily, after an analysis showed an exon 11 mutation.

A new left adrenal gland lesion was seen two years later, and biopsy confirmed it to be a recurrent GIST (Figure 4). He was placed on avapritinib $300 \mathrm{mg}$ daily through a clinical trial with no response and then regorafenib $150 \mathrm{mg}$ daily also with no response. Nevertheless, he underwent resection with negative margins and was given postoperative imatinib $800 \mathrm{mg}$.

One year later, he had a suprarenal recurrence and 


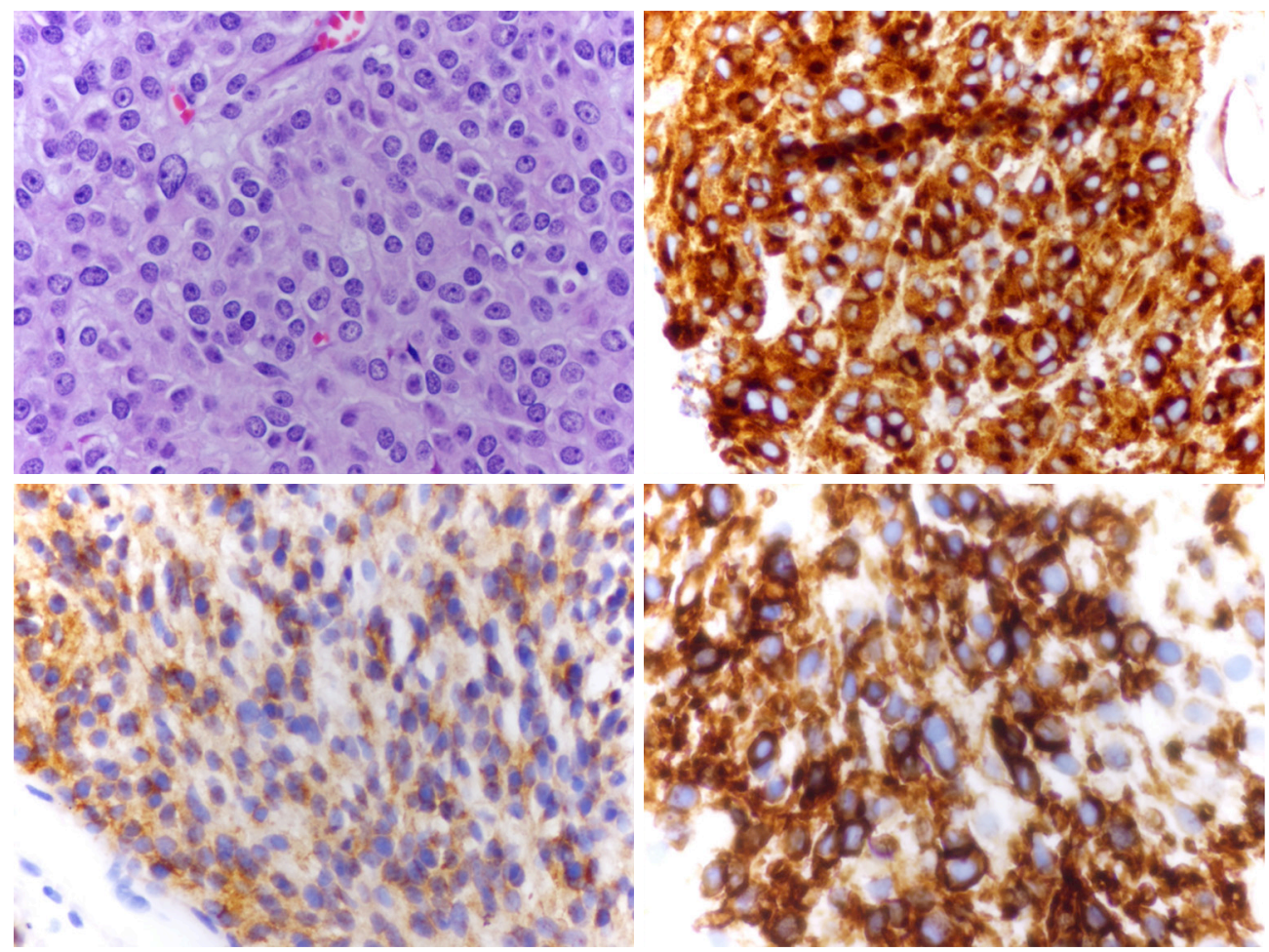

Figure 4 Upper left: Hematoxylin-eosin stain, $\times 40$ original magnification: Gastrointestinal stromal tumor, mixed epithelioid, and spindle cell type. Immunohistochemistry shows that the tumor cells are diffusely strongly positive for CD117 (upper right, $\times 40$ original magnification), DOG1 (lower left, $\times 40$ original magnification), and CD34 (lower right, $\times 60$ original magnification).
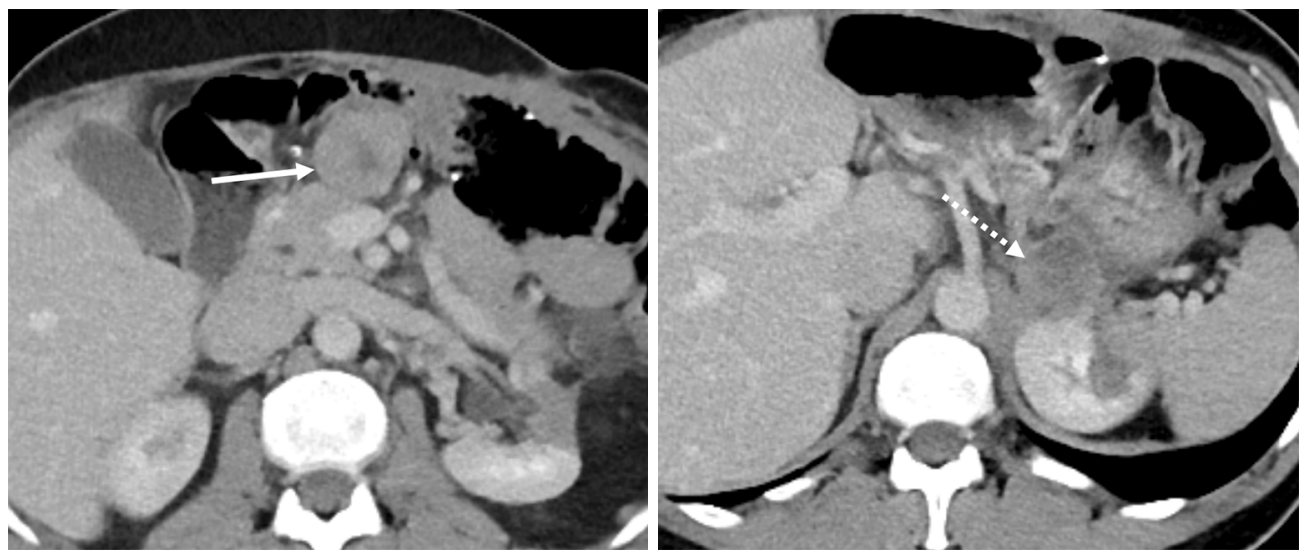

Figure 5 Axial contrast-enhanced CT shows solid peritoneal implants in the anterior upper abdomen (arrow), and anterior to the left kidney (dashed arrow) at the level of the superior mesenteric artery.

development of new masses: one in the upper midline abdomen and one in the retroperitoneum (Figure 5). At that point, patient was started on ripretinib $150 \mathrm{mg}$, but tumor progression continued three months after, with an increase in all the mentioned lesions (Figure 6).
Ripretinib was then escalated to $150 \mathrm{mg}$ twice daily. The patient developed alopecia but otherwise tolerated the higher dose well, with no evidence of new laboratory abnormalities. Three months after dose escalation, a follow-up scan showed an interval decrease in density 

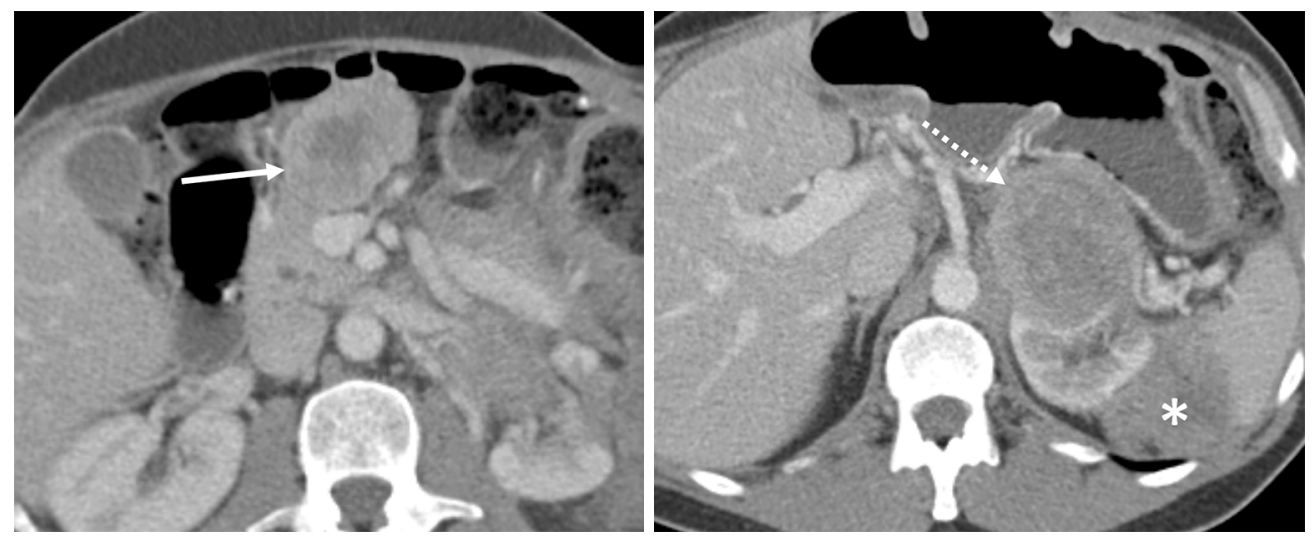

Figure 6 Follow-up CT from three months after demonstrates approximately 35\% interval growth in maximum tumor diameter (from 34 to $46 \mathrm{~mm}$ ) in the anterior upper abdominal mass (arrow) that measured $75 \mathrm{HU}$, and $116 \%$ interval growth in maximum diameter (30 to $65 \mathrm{~mm}$ ) in the pararenal mass (dashed arrow) that measured $60 \mathrm{HU}$. Also, there is a new mass $\left.{ }^{*}\right)$ posterior to the superior pole of the left kidney.
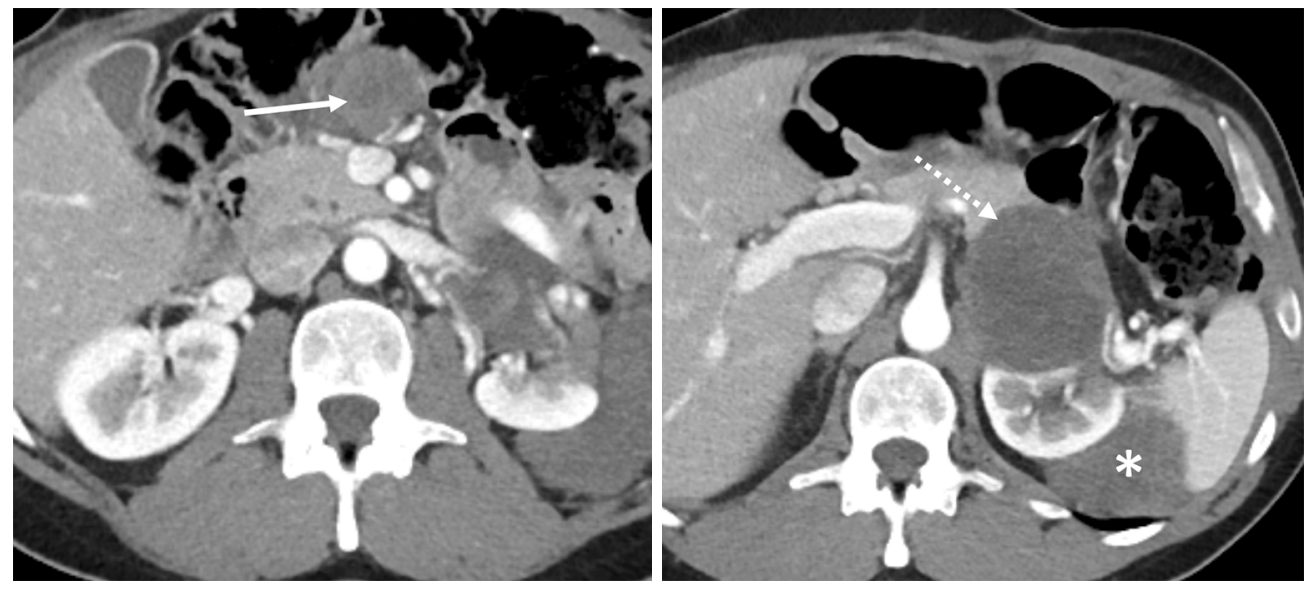

Figure 7 After dose escalation, CT done three months after demonstrates stable size but a 33\% decrease in enhancement in the anterior upper abdominal mass (arrow), and a 50\% decrease in enhancement of the anterior pararenal implant (dashed arrow), constituting partial response by Choi criteria. The mass $\left(^{*}\right)$ posterior the left kidney also shows diminished enhancement.

and size of the upper abdominal and the suprarenal mass, demonstrating response by choi criteria (Figure 7). $\mathrm{He}$ remained progression free for 5 months.

\section{Discussion}

Before the era of tyrosine kinase inhibitors, the management of GIST relied mainly on surgical resection, as GISTs are known to be chemo-resistant (8). The discovery of TKIs was a breakthrough in GIST treatment (8). In 2001, the FDA approved imatinib mesylate for the treatment of metastatic or unresectable GIST. Since then, imatinib is recommended as the first-line treatment for advanced GIST patients, and for adjuvant therapy for high-risk GIST patients by the European Society of Medical Oncology (ESMO), National Comprehensive Cancer Network (NCCN) Task Force, and the Japanese Society of Clinical Oncology (JSCO) (9-11).

Genetic studies have shed light on the biology of GISTs. Now, it is evident that GIST is a genetically heterogenous group of tumors, with distinct clinical characteristics and therapy response. Molecular analysis is essential before initiating treatment, as this will provides the basis not only for surveillance, genetic screening of familial syndromes, but most importantly guide systemic treatment (12). For 
example, NF1 mutated, BRAF mutated and SDH deficient GIST are resistant to imatinib (12). In such subgroup of patients, some treatment strategies include MEK inhibitor trials (NF1 mutated GISTs), BRAF-inhibitors (BRAF mutated GISTs), glutaminase inhibitor trial (SDH deficient GISTs), and guadecitabine trial (SDH deficient GISTs) (12).

Despite initial disease control, advanced GIST patients will develop resistance to imatinib, due to secondary kinase mutations $(4,5)$. Thus, there is a need for drugs that inhibit a broad spectrum of KIT and PDGFRA mutants, inhibiting the resistance mutations and limiting the impact of new mutations (13). In patients with imatinib-resistant disease or who are imatinib-intolerant, sunitinib has a significant clinical benefit with a PFS of 5.6 months $(14,15)$. Regorafenib was studied for patients with progressive disease (PD) on imatinib and sunitinib, resulting in a PFS of 4.8 months $(6,15)$. Other agents that have been investigated in phase II trials include sorafenib (16), pazopanib (17), cabozantinib (18), and dasatinib (19). Despite the current treatment options, there is still an unmet need for further therapies, as the majority of patients will ultimately be refractory to those treatments.

Ripretinib is a type II switch-control TKI that inhibits variety of KIT and PDGFRA mutations, including activating and drug-resistant mutations, as well as other kinases, such as PDGFRB, TIE2, VEGFR2, and BRAF (13). Ripretinib works by forcing the activation loop (or activation switch) into an inactive conformation, hence called switch-control (13). It was approved in 2020 for advanced GIST refractory to at least three TKIs with a standard dose of $150 \mathrm{mg}$ daily (4).

The first-in-human phase I study of ripretinib determined the recommended phase II dose of $150 \mathrm{mg}$ daily, that was associated with a favorable tolerability profile $(4,15)$. In the trial, the maximum dose evaluated was $200 \mathrm{mg}$ twice a day and no maximum tolerated dose was reached (15). Remarkably, ripretinib $150 \mathrm{mg}$ twice daily was also studied and well-tolerated $(4,15)$. Only three patients out of 258 experienced dose-limiting toxicity in the study: Two had an asymptomatic grade 3 lipase elevations, and one, an asymptomatic grade 4 creatine phosphokinase elevation (15). Neither of the patients described here experienced limiting side effects, including elevations in lipase or creatine phosphokinase.

Ripretinib most common side effects include alopecia, myalgia, nausea, fatigue, and hand-foot syndrome $(4,15)$. Only case 2 experienced alopecia and fatigue. The pathogenesis of the ripretinib induced alopecia is unclear but may be due to the inhibition alopecia associated kinases. Case 1 experienced hand-foot syndrome while on regorafenib, but not on ripretinib. Alopecia has been reported with other TKIs, but it seems higher with ripretinib (15). Similarly, hand-foot syndrome (reported in the trials as palmar-plantar erythrodysesthesia) is reported in the phase I trial in $43.7 \%$ of patients receiving ripretinib $150 \mathrm{mg}$ daily and only one patient experienced as grade 3 $(0.7 \%)(15)$. To put into perspective, hand-foot syndromes have been reported as grade 3 in $4 \%$ and $19.7 \%$ with sunitinib and regorafenib, respectively (15).

The two cases we described progressed while receiving the standard dose of ripretinib but responded after dose escalation. In both trials (the INVICTUS trial and phase I trial), patients receiving ripretinib at $150 \mathrm{mg}$ daily with $\mathrm{PD}$ were allowed to dose escalate to $150 \mathrm{mg}$ twice a day $(4,15)$. Sub-analysis of the phase I trial showed that 64 patients ultimately received ripretinib at $150 \mathrm{mg}$ twice daily. They achieved a PFS of 8.3 months when ripretinib $150 \mathrm{mg}$ twice a day was used as second or third line therapy, and 5.5 months when used as fourth line, with a similar safety profile compared to $150 \mathrm{mg}$ daily (20). Like imatinib, where dose escalation is a valid strategy after PD in the standard dose (21), ripretinib dose-escalation also seems to be a suitable strategy. Our cases illustrate that patients that progress under standard dose ripretinib can not only achieve stable disease but also radiological responses with dose escalation. To minimize the possibility of an initial pseudo-progression, we analyzed the images utilizing Choi criteria that considers density changes (7).

Since the PFS of ripretinib exceeds those historically reported for second-line sunitinib therapy (5.6 months) and third-line regorafenib therapy (4.8 months), investigators are now conducting a phase III study (INTRIGUE; NCT03673501) in which ripretinib is being compared to sunitinib as second-line therapy. Future studies are needed to assess the optimal strategy of the dose escalation of ripretinib; after progression on the standard dose, as a ripretinib re-challenge after a different agent or even initially in a subset of patients harboring specific mutations or clinical characteristics.

\section{Conclusions}

Dose escalation of ripretinib is well-tolerated and leads to objective responses in patients whose tumors progressed after standard dose. Future studies are needed to establish 
when this strategy should be best used.

\section{Acknowledgments}

Funding: This work was supported by the National Cancer Institute at the National Institutes of Health [P30CA240139 to JCT].

\section{Footnote}

Reporting Checklist: The authors have completed the CARE reporting checklist. Available at http://dx.doi.org/10.21037/ gist-21-1

Conflicts of Interest: All authors have completed the ICMJE uniform disclosure form (available at http://dx.doi. org/10.21037/gist-21-1). GD is a Deciphera's advisor board consultant. JCT is consultant for Deciphera, Bayer, Daiichi, Epizyme, Blueprint and C4 Therapeutics. The other authors have no conflicts of interest to declare.

Etbical Statement: The authors are accountable for all aspects of the work in ensuring that questions related to the accuracy or integrity of any part of the work are appropriately investigated and resolved. All procedures performed in studies involving human participants were in accordance with the ethical standards of the institutional and/or national research committee(s) and with the Helsinki Declaration (as revised in 2013). Written informed consent was obtained from the patient.

Open Access Statement: This is an Open Access article distributed in accordance with the Creative Commons Attribution-NonCommercial-NoDerivs 4.0 International License (CC BY-NC-ND 4.0), which permits the noncommercial replication and distribution of the article with the strict proviso that no changes or edits are made and the original work is properly cited (including links to both the formal publication through the relevant DOI and the license). See: https://creativecommons.org/licenses/by-nc-nd/4.0/.

\section{References}

1. Florou V, Trent JC, Wilky BA. Precision medicine in gastrointestinal stromal tumors. Discov Med 2019;28:267-76.

2. Hirota S, Isozaki K, Moriyama Y, et al. Gain-of-function mutations of c-kit in human gastrointestinal stromal tumors. Science 1998;279:577-80.

3. Heinrich MC, Corless CL, Duensing A, et al. PDGFRA activating mutations in gastrointestinal stromal tumors. Science 2003;299:708-10.

4. Blay JY, Serrano C, Heinrich MC, et al. Ripretinib in patients with advanced gastrointestinal stromal tumours (INVICTUS): a double-blind, randomised, placebocontrolled, phase 3 trial. Lancet Oncol 2020;21:923-34.

5. Heinrich MC, Corless CL, Blanke CD, et al. Molecular Correlates of Imatinib Resistance in Gastrointestinal Stromal Tumors. J Clin Oncol 2006;24:4764-74.

6. Demetri GD, Reichardt P, Kang YK, et al. Efficacy and safety of regorafenib for advanced gastrointestinal stromal tumours after failure of imatinib and sunitinib (GRID): an international, multicentre, randomised, placebocontrolled, phase 3 trial. Lancet 2013;381:295-302.

7. Arshad J, Ahmed J, Subhawong T, et al. Progress in determining response to treatment in gastrointestinal stromal tumor. Expert Rev Anticancer Ther 2020;20:279-88.

8. Trent JC, Beach J, Burgess MA, et al. A two-arm phase II study of temozolomide in patients with advanced gastrointestinal stromal tumors and other soft tissue sarcomas. Cancer 2003;98:2693-9.

9. Demetri GD, von Mehren M, Antonescu CR, et al. NCCN Task Force report: update on the management of patients with gastrointestinal stromal tumors. J Natl Compr Canc Netw 2010;8 Suppl 2:S1-41; quiz S2-4.

10. Casali PG, Abecassis N, Aro HT, et al. Gastrointestinal stromal tumours: ESMO-EURACAN Clinical Practice Guidelines for diagnosis, treatment and follow-up. Ann Oncol 2018;29:iv267.

11. Nishida T, Hirota S, Yanagisawa A, et al. Clinical practice guidelines for gastrointestinal stromal tumor (GIST) in Japan: English version. Int J Clin Oncol 2008;13:416-30.

12. Mei L, Smith SC, Faber AC, et al. Gastrointestinal Stromal Tumors: The GIST of Precision Medicine. Trends Cancer 2018;4:74-91.

13. Dhillon S. Ripretinib: First Approval. Drugs 2020;80:1133-8.

14. Demetri GD, van Oosterom AT, Garrett CR, et al. Efficacy and safety of sunitinib in patients with advanced gastrointestinal stromal tumour after failure of imatinib: a randomised controlled trial. Lancet 2006;368:1329-38.

15. Janku F, Abdul Razak AR, Chi P, et al. Switch Control Inhibition of KIT and PDGFRA in Patients With Advanced Gastrointestinal Stromal Tumor: A Phase I Study of Ripretinib. J Clin Oncol 2020;38:3294-303. 
16. Park SH, Ryu MH, Ryoo BY, et al. Sorafenib in patients with metastatic gastrointestinal stromal tumors who failed two or more prior tyrosine kinase inhibitors: a phase II study of Korean gastrointestinal stromal tumors study group. Invest New Drugs 2012;30:2377-83.

17. Ganjoo KN, Villalobos VM, Kamaya A, et al. A multicenter phase II study of pazopanib in patients with advanced gastrointestinal stromal tumors (GIST) following failure of at least imatinib and sunitinib. Ann Oncol 2014;25:236-40.

18. Schoffski P, Mir O, Kasper B, et al. Activity and safety of the multi-target tyrosine kinase inhibitor cabozantinib in patients with metastatic gastrointestinal stromal tumour after treatment with imatinib and sunitinib: European

\section{doi: 10.21037 /gist-21-1}

Cite this article as: Costa PA, Hana CKA, Balaji NC, Skryd AF, Valdes BN, Minjares RO, Barreto-Coelho P, EspejoFreire AP, Hakim MO, Jonczak E, Subhawong T, Livingstone A, Trent JC, D'Amato GZ. Dose escalation of ripretinib can lead to response in advanced gastrointestinal stromal tumor patients refractory to the standard dose: a report of two cases. Gastrointest Stromal Tumor 2021;4:1.
Organisation for Research and Treatment of Cancer phase II trial 1317 'CaboGIST'. Eur J Cancer 2020;134:62-74.

19. Li J, Zhou Y, Zhang X, et al. Safety and efficacy of dasatinib in patients with advanced gastrointestinal stromal tumors refractory to imatinib and sunitinib: A single arm, multicenters, phase 2 trial. J Clin Oncol 2019;37:138.

20. Janku F, Chi P, Heinrich M, et al. 1623MO Ripretinib intra-patient dose escalation (IPDE) following disease progression provides clinically meaningful progressionfree survival (PFS) in gastrointestinal stromal tumor (GIST) in phase I study. Ann Oncol 2020;31:S974-5.

21. Verweij J, Casali PG, Zalcberg J, et al. Progression-free survival in gastrointestinal stromal tumours with high-dose imatinib: randomised trial. Lancet 2004;364:1127-34. 